\section{Mistura Enantiomérica da Bupivacaína, a Obviedade Estereoisomérica}

Senhor Editor,

No último número de 2002 da Revista Brasileira de Anestesiologia, Soares e col. ${ }^{1}$, num estudo muito bem conduzido em pacientes oftalmológicos, investigaram a Mistura Enantiomérica da Bupivacaína. Este estudo, é o primeiro em que a Mistura Enantiomérica da Bupivacaína foi utilizada na anestesia ocular. Todavia, notamos que na discussão, passou desapercebida a impropriedade da citação bibliográfica de número 13 , fato que confirma a confusão terminológica reinante.

Esta referência ${ }^{2}$ trata-se da pesquisa básica de nossa autoria, no modelo nervo ciático de rato, onde comparamos a levobupivacaína com a bupivacaína, quanto à atividade bloqueadora de nervo. Aimpropriedade da citação deveu-se ao fato dos Autores justificarem a ausência de toxicidade da Mistura Enantiomérica da Bupivacaína, valendo-se de uma pesquisa que absolutamente não aborda aspectos toxicológicos enquanto outro agente foi estudado, diferente da Mistura Enantiomérica da Bupivacaína.

Embora que a citação ao nosso trabalho tenha sido lisonjeira, urge uma correção e esperamos que os Autores aceitem esta nossa observação. Aguisa de colaboração sugerimos a leitura do trabalho de Delfino e col. ${ }^{3}$, que aborda com muita precisão os conceitos enantioméricos que redundaram na Mistura Enantiomérica da Bupivacaína. Ainda, mencionamos os nossos estudos na fase pré-clínica, que versam sobre cárdio e neurotoxicidade deste novo anestésico local ${ }^{4-6}$. No primeiro estudo investigamos a toxicidade potencial de anestésicos locais inclusive do isômero dextrógiro no rato in vivo, comparados com a Mistura Enantiomérica da Bupivacaína. Nesta pesquisa, concluímos que a cardiotoxicidade potencial da Mistura Enantiomérica da Bupivacaína (S75:R25 e/ou S90:R10) é igual a da levobupivacaína. Portanto, a despeito de conter o isômero $\mathrm{R}(+)$ ou dextrógiro ou ainda "isômero mau", em proporções reduzidas, esses racemados demonstram eficácia no bloqueio do nervo, enquanto a toxicidade potencial não é aumentada, o que lhe confere a segurança que não existe na bupivacaína racêmica (S50:R50). Seguramente, este achado justifica a vantagem da Mistura Enantiomérica da Bupivacaína, um composto taIhado isomericamente visando a obtenção de índice terapêutico favorável.

O novo anestésico local brasileiro, ou ex-Simocaína, nasceu de uma especulação científica que nos fez realizar uma obviedade estereoisomérica, resultando no binômio Eficácia/Segurança. E fizemos antes que alguém no mundo, o fizesse.

Outro problema que gostaria de ressaltar é que mesmo assim, está havendo imprecisões nominais para designar este novo agente na nossa comunidade. Senão vejamos:
Poucos diferenciam a Levobupivacaína da Mistura Enantiomérica da Bupivacaína. Numa pesquisa que realizamos em diferentes encontros científicos, pudemos captar a confusão reinante entre os colegas quanto à terminologia.

Qualquer que seja o nome que se venha dar ao composto, fruto da "modificação estereoisomeria baseada em evidência", o racemado específico vem se consagrando graças aos trabalhos clínicos publicados pela Revista Brasileira de Anestesiologia, que os Autores chamam-no de "Mistura Enantiomérica da Bupivacaína ${ }^{1,2,7,8}$.

Deve ficar claro que a bupivacaína lançada com o nome de Marcaína ${ }^{\circledR}$ pela Astra Química, apresenta relação equimolar de 1:1. A levobupivacaína (patente pertencente a Chiroscience), que está chegando ao Brasil através do Laboratório Abbott, é a forma levógira pura. A Mistura Enantiomérica de Bupivacaína tem a relação S75-R25 e está sendo comercializada pela Cristália com o nome de Novabupi ${ }^{\circledR}$.

Maria P. B. Simonetti, TSA

Rua Jacurici, 166/ 112 Bloco 1 - Itaim

01453-000 São Paulo, SP

E-mail: simonett@icb.usp.br

\section{Enantiomeric Bupivacaine Mixture, the Obvious Nature of Stereoisomism}

In the last 2002 edition of the Brazilian Journal of Anesthesiology, Soares et al. ${ }^{1}$, in a well-conducted study with ophthalmologic patients, have investigated Enantiomeric Bupivacaine Mixture. This is the first study in which Enantiomeric Bupivacaine Mixture is used in ocular anesthesia. However, we have noticed that in the discussion, the impropriety of reference number 13 has gone unnoticed, thus confirming current terminological confusion.

This reference ${ }^{2}$ is our basic research with sciatic nerve of rats where nervous blockade activity of levobupivacaine and bupivacaine is compared. The impropriety of the reference resides in the fact that the authors have justified the absence of Enantiomeric Bupivacaine Mixture toxicity based on a research which does not approach toxicological aspects whatsoever and has studied an agent different from Enantiomeric Bupivacaine Mixture.

Although the reference to our work was flattering, a correction is necessary and we hope that the Authors accept this observation. As cooperation, we suggest the reading of Delfino et al. ${ }^{3}$ work, which very accurately addresses enantiomeric concepts resulting in Enantiomeric Bupivacaine Mixture. We also mention our preclinical studies on cardio and neurotoxicity of this new local anesthetic agent ${ }^{4-6}$.

In the first study, we have investigated the potential toxicity of local anesthetics, including the dextrogyrous isomer, as compared to Enantiomeric Bupivacaine Mixture, in in vivo 
studies with rats. In this research, our conclusion was that the potential cardiotoxicity of Enantiomeric Bupivacaine Mixture (S75:R25 and/or S90:R10) was equal to levobupivacaine. So, although containing the $\mathrm{R}(+)$ isomer, or dextrogyrous, or even the "bad isomer" in small amounts, these racemic agents are effective for nervous block and potential toxicity is not increased, giving them the safety which is absent in racemic bupivacaine (S50:R50). Clearly, these findings justify the advantage of Enantiomeric Bupivacaine Mixture, an isomeric compound aiming at reaching a favorable therapeutic index.

The new Brazilian local anesthetic agent, or former-Simocaine, was born after a scientific speculation that made us realize a stereoisomeric reality, resulting in the Efficacy/Safety binomial. And we did it before anyone else in the world would do it.

Another issue we would like to highlight is that, even so, there are nominal incorrectnesses in defining this agent within our community:

Few differentiate levobupivacaine from Enatiomeric Bupivacaine Mixture. In a survey we carried out during different scientific meetings, we could capture this terminological confusion among colleagues.

Whatever the name given to the compound, fruit of the "evidence-based stereoisomeric modification" the specific racemic agent is being acclaimed thanks to clinical studies published by the Brazilian Journal of Anesthesiology, and the Authors call it Enantiomeric Bupivacaine Mixture ${ }^{1,2,7,8}$.

It has to be made clear that bupivacaine, introduced with the name Marcaína ${ }^{\circledR}$ by Astra Química, has an equimolar 1:1 ratio, levobupivacaine (patent belonging to Chiroscience), is being brought to Brazil by Abbott Laboratories, and is the pure levogyrous form. Enantiomeric Bupivacaine Mixture has an S75-R25 ratio and is being marketed by Cristalia with the name Novabupi ${ }^{\circledR}$.

Maria P. B. Simonetti, TSA, M.D.

Rua Jacurici, 166/ 112 Bloco 1 - Itaim 01453-000 São Paulo, SP

E-mail: simonett@icb.usp.br

\section{REFERÊNCIAS - REFERENCES}

01. Soares LF, Helayel PE e al - Bloqueio peribulbar com a associação da mistura enantiomérica de bupivacaína (S75 R25) a 0,5\% e lidocaína a 2\%: efeitos da adição de hialuronidase. Rev Bras Anestesiol, 2002;52:420-425.

02. Simonetti MPB, Valinetti EA - Avaliação da atividade anestésica local da S(-) bupivacaína: estudo experimental in vivo no nervo ciático de rato. Rev Bras Anestesiol, 1997;47:425-434.

03. Delfino J, Vale N - Bupivacaína levógira a $0,5 \%$ pura versus mistura enantiomérica de bupivacaína (S75-R25) a 0,5\% em anestesia peridural para cirurgia de varizes. Rev Bras Anestesiol, 2001;51:474-482.
04. Simonetti MPB, Ferreira FMC - Obtenção de novos anestésicos locais através da modificação da relação enantiomérica da bupivacaína racêmica: Rev Bras Anestesiol, 1999;49:(Supl): CBA156.

05. Simonetti MPB - Optimization of the therapeutic index of bupivacaine through the manipulation of the enantiomeric ratio, $12^{\text {th }}$ World Congress of Anaesthesiologists, Canada, 2000; P6.05.

06. Simonetti MPB, Bird RA - Evaluation of new local anesthetics obtained through the manipulation of the enantiomeric ratio of bupivacaine on the central nervous system of the rat. The International Monitor, ESRA, 2000;12:129.

07. Imbelloni LE, Beato L - Comparação entre bupivacaína racêmica (S50-R50) e mistura enantiomérica de bupivacaína (S75-R25), ambas isobáricas, a $0,5 \%$ em raquianestesia. Estudo em cirurgias ortopédicas, Rev Bras Anestesiol, 2001; 51:369-376.

08. Imbelloni LE, Vieira EM - Raquianestesia com a mistura enantiomérica de bupivacaína a 0,5\% isobárica (S75-R25) em crianças com idade de 1 a 5 anos para cirurgia ambulatorial, Rev Bras Anestesiol, 2002;52:286-293.

\section{Material Inadequado para Raqui- anestesia. Até Quando?}

Senhor Editor,

Os tumores epidermóides do canal medular são extremamentes raros, não existindo qualquer relato desta complicação na Revista Brasileira de Anestesiologia. Já estamos em pleno século XXI, e o uso de material descartável para a raquianestesia é inquestionável ${ }^{1}$. Entretanto, muitos anestesiologistas continuam empregando material inadequado ou fazendo bloqueio de nervos periféricos com agulhas hipodérmicas para injeções musculares e venosas. Para penetrar no sistema nervoso no neuro-eixo é necessário passar por oito barreiras antes de se obter LCR que são: 1) pele; 2) tecido celular subcutâneo; 3) ligamento supra-espinhal; 4) ligamento interespinhal; 5) ligamento amarelo; 6) espaço extradural; 7) duramáter; 8) aracnóide-mater; e, 9) espaço leptomeníngeo ${ }^{2}$. Aagulha ideal de raquianestesia deve ser descartável, facilitar a identificação do espaço subaracnóideo e a injeção do anestésico local, não se deformar, não causar outras doenças, além de possuir mandril. O material de raquianestesia é classificado como crítico ${ }^{1}$. É, portanto, de alto risco e por isso deve ser de uso único ${ }^{1}$. Em editorial de $1977^{3}$ acreditava-se que os tumores epidermóides tenderiam a desaparecer com o uso de agulhas descartáveis.

Tumores epidermóides espinhais são raros. Podem ser congênitos ou iatrogênicos. Os iatrogênicos originam-se da implantação de células epiteliais dentro do canal espinhal durante punções lombares diagnósticas ${ }^{4-6}$, terapêuticas ${ }^{7-9}$, exames de mielografias ${ }^{10}$, cirurgia de coluna, em particular na correção de meningoceles ${ }^{11}$, nos acidentes com armas de fogo ${ }^{12}$ e após anestesias praticadas na raquianestesia 
9,13,14. Estas complicações podem acontecer se não utilizarmos material de punção adequado. Em revisão de 90 casos de tumores epidermóides realizada em 1962, 41\% foram causados por iatrogenia de diferentes causas ${ }^{13}$. Reina e col. em 1996, mostraram mais de 100 casos publicados na bibliografia de tumores epidermóides de causa iatrogênica ${ }^{15}$, sendo que de 1977 a 1995 foram comunicados 29 novos casos ${ }^{15}$.

O tumor epidermóide iatrogênico é resultado de punções lombares ou caudais realizadas com agulhas sem mandril ou com agulhas com mandril inadaptados. A punção da pele, com agulhas inadequadas carreia fragmentos epidérmicos para dentro do canal espinhal ${ }^{16}$. Ao analisar punções em cadáveres, com agulhas peridural e raquidiana, foi observada incidência de fragmentos epidérmicos de $45 \%$ com Tuohy $16 \mathrm{G}$ e $30 \%$ com Tuohy $17 \mathrm{G} ; 15 \%$ com Quincke $22 \mathrm{G}$ e $30 \%$ com Sprotte 22G ${ }^{17}$. Da mesma forma, estudo com cânulas de punção venosa empregadas para bloqueio caudal em 50 crianças, demonstrou tecido epidermóide em 33\%, tecido gorduroso em $67 \%$ e sangue em $26 \%$ dos casos ${ }^{18}$. O desenvolvimento de tumores iatrogênicos foi demonstrado experimentalmente por diferentes grupos de investigadores ${ }^{19,20}$. O uso de escalpe foi proposto para facilitar as punções lombares diagnósticas em neonatos com objetivo de simplificação da técnica ${ }^{21}$. Esta sugestão teve muita aceitação entre os neonatologistas, mas foi bastante criticada por outros autores $^{22,23}$, que a consideraram muito perigosa pela possibilidade de introdução de fragmentos epiteliais dentro do canal espinhal.

O período de silêncio até apresentação de sintomas pode durar de 2 a 10 anos. O intervalo mais comum gira entre 4 e 8 anos, embora existam casos com até 20 e 41 anos de intervalo entre a causa e os sintomas ${ }^{15}$. Alocalização dos implantes de fragmentos cutâneos é preferencialmente na região lombar, mas pode aparecer no nível torácico ou sacral. O seu prognóstico é bom e o tratamento é cirúrgico. Se o tumor for ressecado sem complicações, o paciente pode recuperar progressivamente o deficit motor.

Reina e col. concluíram seu trabalho de revisão afirmando que "atualmente, considerando todos os tumores epidermóides iatrogênicos comunicados, pode-se interpretar como uma conduta imprudente o uso de agulhas sem mandril para realizar punções lombares pelo potencial problema que podem ocasionar" ${ }^{15}$. Alves Neto e col., em Anestesia e Bioética, terminou dizendo que a solução é lançar mão da tolerância e, principalmente a prudência para assegurar o respeito à autonomia do paciente ${ }^{24}$

Luiz Eduardo Imbelloni, TSA

M. A. Gouveia, TSA

Elaine Aparecida Félix Fortis, TSA

Av. Epitácio Pessoa, 2356/203

22471-000 - Rio de Janeiro, RJ

\section{Inadequate Spinal Anesthesia Material. Until When?}

\author{
Mr. Editor,
}

Epidermoid spinal cord tumors are extremely rare with no reports of such complication so far published in the Brazilian Journal of Anesthesiology. We are already in the $21^{\text {st }}$ century and the use of disposable material is unquestionable ${ }^{1}$.

Many anesthesiologists however are still using inadequate material or blocking peripheral nerves with muscular or intravenous injection needles. To penetrate the nervous system at the neuro-axis it is necessary to overcome nine barriers before obtaining CSF: 1) skin; 2) subcutaneous tissue; 3) supra-spinal ligament; 4) inter-spinal ligament; 5) yellow ligament; 6) extradural space; 7) duramater; 8) arachnoid-mater; and 9) leptomeningeal space ${ }^{2}$. The ideal spinal anesthesia needle should be disposable, help identifying spinal space and local anesthetic injection, and should not deform, not cause other diseases, in addition to having a mandrel. Spinal anesthesia material is classified as critical ${ }^{1}$. It is then of high risk and should be used only once ${ }^{1}$. In an editorial of $1977^{3}$, it was believed that epidermoid tumors would tend to disappear with the use of disposable needles.

Epidermoid spinal tumors are rare and may be congenital or iatrogenic. latrogenic tumors originate from the implantation of epithelial cells within the spinal cord during diagnostic ${ }^{4-6}$ or therapeutic ${ }^{7-9}$ lumbar punctures, myelography ${ }^{10}$, spinal surgeries, especially meningocele correction ${ }^{11}$, gunshot accidents ${ }^{12}$ and after spinal anesthesia ${ }^{9,13,14}$. These complications may be present if inadequate puncture material is used. In a review of 90 epidermoid tumors, performed in 1962, 41\% of them were caused by different iatrogenies ${ }^{13}$. Reina et al., in 1996, have shown more than 100 epidermoid iatrogenic tumors published in the literature ${ }^{15}$, being 29 new cases informed from 1977 to $1995^{15}$.

Epidermoid iatrogenic tumors are result of lumbar or caudal punctures performed with mandrel-less needles or with unadapted mandrels. Skin puncture with inadequate needles takes skin fragments to inside the spinal cord ${ }^{16}$. In analyzing cadaver punctures with epidural and spinal needles it was observed $45 \%$ of skin fragments with 16 G Tuohy and $30 \%$ with 17G Tuohy needles; $15 \%$ with 22G Quincke and 30\% with $22 \mathrm{G}$ Sprotte needles ${ }^{17}$. Similarly, a study with intravenous puncture cannulas used for caudal block in 50 children, has shown epidermoid tissue in 33\%, fatty tissue in $67 \%$ and blood in $26 \%$ of cases ${ }^{18}$. The development of iatrogenic tumors has been experimentally shown by different researchers ${ }^{19,20}$. Scalps were proposed to help diagnostic lumbar punctures in neonates, aiming at technique simplification ${ }^{21}$. This has been widely accepted by neonatologists, but was widely criticized by other authors ${ }^{22,23}$ who consider it very dangerous for the possibility of introducing epithelial fragments in the spinal cord.

The silent period until symptoms presentation may last 2 to 10 years. The most common interval is around 4 and 8 years, 
although there are cases with up to 20 and 41 -year intervals between cause and symptoms ${ }^{15}$. Skin fragments are preferably located in the lumbar region, but they may appear at thoracic or sacral levels. Prognosis is favorable and treatment is surgical. If the tumor is resected without complications, patient may progressively recover from motor deficit.

Reina et al. have concluded their review by stating that "currently, considering all communicated epidermoid iatrogenic tumors, one may consider imprudent the use of mandrel-less needles for lumbar punctures due to potential problems they may bring" ${ }^{15}$. Alves Neto et al., in Anesthesia and Bioethics, ended up stating that the solution is to use tolerance and moreover prudence to assure the respect to patients' autonomy ${ }^{24}$.

Yours Truly.

Luiz Eduardo Imbelloni, TSA, M.D.

M. A. Gouveia, TSA, M.D.

Elaine Aparecida Félix Fortis, TSA, M.D.

Av. Epitácio Pessoa, 2356/203

22471-000 Rio de Janeiro, RJ

\section{REFERÊNCIAS - REFERENCES}

01. Imbelloni LE, Fortis EF - Agulhas, Catéteres, Técnicas e Drogas, em: Imbelloni LE - Tratado de Anestesia Raquidiana, Medidática Informática Ltda, Curitiba, 2001;6:57-66.

02. Imbelloni LE, Katayama M - Bloqueio subaracnóideo. Rev Bras Anestesiol, 1995;45: (Supl20):76-80 .

03. Lumbar puncture and epidermoid tumor. Lancet, 1977;1:635 ( $\mathrm{n}^{\circ}$ 8012).

04. Halcrow SJ, Crawford PJ, Craft AW - Epidermoid spinal cord tumour after lumbar puncture. Arch Dis Childhood, 1985;60: 978-979.

05. Blockey NJ, Schorstein J - Intraspinal epidermoid tumour in the lumbar region of children. J Bone Joint Surg, 1961;43B: 556-562.

06. Demaerel P, Casaer P, van Calenbergh F et al - A iatrogenic spinal epidermoid tumour associated with tuberous sclerosis. A diagnostic pitfall. J Neuroradiol, 1994;21:273-273.

07. Lasierra AP, Gutiérrez PR - Sobre los Ilamados colesteatomas de inclusión. Rev Clin Esp, 1959;74:242-246.

08. Kohama Y, Imamura H, Hida K et al - A case of spinal epidermoid caused by lumbar punctures. Neurological Surgery, 1996;24: 375-378.

09. Blázquez MG, Oliver B - Epidermoides intrarraquídeos de inclusion iatrogénica. Arch Neurobiol, 1980;43:217-228.

10. Gardner RC - Intradural cholesteatoma (pearly tumor): a rare complication of myelography or spinal puncture. Southern Med J, 1973;66:1070-1071.

11. Kirsch WM, Hodges FJ - An intramedullary inclusion cyst of the thoracic cord associated with a previously repaired meningocele. Case report. J Neurosurg, 1966;24:1018-1020.

12. Smith CM, Timperley WR - Multiple intraspinal and intracranial epidermoids and lipomata following gunshot injury. Neuropathol Appl Neurobiol, 1984;10:235-239.

13. Manno NJ, Uihlein A, Kernohan J - Intraspinal epidermoids. J Neurosurg, 1962;19:754-765.

Revista Brasileira de Anestesiologia

Vol. 53, № 2, Março - Abril, 2003
14. Mac Donald JV, Klump TE - Intraspinal epidermoid tumors caused by lumbar puncture. Arch Neurol 1986;43:423-424.

15. Reina MA, López-García A, Dittmann $M$ et al - Tumores epidermoides espinales iatrogénicos. Una complicación tardía de la punción espinal. Rev Esp Anestesiol Reanim, 1996;43: 142-146.

16. Di Giovanni AJ - A critical evaluation of disposable spinal anesthesia leedles. Anesthesiology, 1971;34:88-92.

17. Reina MA, López-Garcia A, Manzarbeitia F et al - Arrastre de fragmentos epidérmicos mediante agujas espinales em cadáveres. Rev Esp Anestesiol Reanim, 1995;42:383-385.

18. Goldchneider KR, Brandom BW - The incidence of tissue coring during the performance of caudal injection in children. Reg Anesth Pain Med, 1999;24:553-556.

19. Van Gilder JG, Schwartz HG - Growth of dermoids from skin implants to the nervous system and surrounding spaces of the newborn rat. J Neurosurg, 1967;26:14-20.

20. Oblu N, Wasserman L, Sandulescu G et al - Experimental investigation of the origin of intraspinal epidermoid cysts. Acta Neurol Scandinav 1967;43:79-86.

21. Greensher J, Mofenson JC, Borofkly LG et al - Lumbar puncture in the neonate: A simplified technique. J Pediatr, 1971;78:1034.

22. Shaywitz BA - Epidermoid spinal cord tumors and previous lumbar punctures. J Pediatr, 1972;80:638-640.

23. Batnizky S, Keucher TR, Mealey J et al - latrogenic intraspinal epidermoid tumors. JAMA, 1977;237:148-450.

25. Alves Neto O, Garrafa V - Anestesia e bioética. Rev Bras Anestesiol, 2000;50:178-188.

\section{Agulhas com Mandril ou Agulhas Comuns para o Bloqueio Caudal na Criança?}

Senhor Editor,

A punção do hiato sacro para a realização de anestesia caudal em criança sempre foi feita com agulhas comuns de injeção. Esta prática persiste a despeito de haver hoje disponíveis agulhas com mandril apropriadas ao uso pediátrico. Geiduschek ${ }^{1}$ reconhece que: embora alguns produtos tenham sido desenhados especialmente para anestesia regional na criança, o bloqueio caudal pode serfeito com agulhas $20 \mathrm{G} \mathrm{ou}$ $22 \mathrm{G}$ simples ou com cateter venoso. Recentemente no entanto o uso de agulhas sem mandril no bloqueio caudal vem sendo criticado por alguns autores como Dalens ${ }^{2}$, Broad$\mathrm{man}^{3} \mathrm{e}$ Tobias ${ }^{4}$ por acreditarem que estas agulhas podem carrear em seu interior células epiteliais que conduzidas até a medula espinhal poderiam formar tumores epidermóides. Broadman ${ }^{3}$, em longo editorial, utiliza 23 referências para embasar sua posição contra o uso das agulhas sem mandril nos bloqueios sacros. Ameu ver ele não apresenta argumentos convincentes. Reconhece por exemplo que: a) com uma única exceção, todos os relatos de tumores epidermóides publicados na literatura ocorreram no espaço intradural em pacientes que haviam recebido anestesia raquidiana; b) em nenhum destes casos foi estabelecida relação de causa-efe- 
ito entre a punção e o surgimento do epitelioma; c) a presença de mandril na agulha não é garantia de que estas não carrearão células epiteliais em seu interior, conforme citação de pesquisa feita com cadáveres; neste estudo células epiteliais foram encontradas dentro de agulhas Tuohy 16G, Sprotte $22 \mathrm{G}$ e Quincke 22G. Resultado parecido foi obtido em seres humanos submetidos à anestesia raquidiana com agulha $25 \mathrm{G}$ com mandril ${ }^{5}$; d) não se sabe ao certo ainda se os tumores epidermóides são formados a partir das células transportadas pelas agulhas ou de células embrionárias.

Embora algumas autoridades, certamente bem intencionadas, condenem de modo incisivo a realização de anestesia caudal com agulhas comuns, pesquisadores igualmente respeitados continuam a utilizar estas agulhas em seus bloqueios sacros. Apenas para citar algumas publicações recentes. Um grupo francês utilizou agulhas $22 \mathrm{G}$ para anestesia caudal como técnica única em neonatos ${ }^{6}$; Anestesiologistas canadenses introduziram catéteres pelo hiato sacro através de agulhas de injeção venosa $18 \mathrm{G}^{7}$. Aúltima edição do livro editado por George Gregory, referência mundial em anestesia pediátrica, recomenda que anestesia caudal seja feita com agulhas hipodérmicas ${ }^{8}$. Mesma conduta está preconizada no compêndio editado por Morgan e col. ${ }^{9}$. Coté e co-editores ofereceram as duas opções: agulhas com mandril ou agulhas para punção venosa com cateter ${ }^{10}$.

Se não há consenso na literatura porque não mudarmos então para as agulhas com mandril? Por dois motivos: o preço e a disponibilidade das agulhas com mandril. Agulhas comuns custam centenas de vezes menos que agulhas com mandril e podem ser encontradas em qualquer hospital. O próprio Bradman ${ }^{3}$ reconhece em seu editorial que: sempre que o tamanho do paciente permitir o uso de uma agulha com introdutor, devemos empregá-lo. Entende-se que nem ele dispõe de agulhas com mandril para todo tamanho do paciente! $\mathrm{E}$ neste caso fazer o que? Utilizar uma agulha comum ou não fazer o bloqueio sacro são as únicas alternativas. Será que não fazer um bloqueio sacro, quando indicado, não traria maior risco para o paciente do que usar uma agulha comum? A anestesia caudal é de extrema utilidade na criança. Sua técnica é simples, reduz a necessidade de drogas anestésicas e relaxantes musculares durante a cirurgia e provê analgesia pós-operatória. Certamente tem seus riscos mas a Medicina sem riscos não existe! Não há dúvidas de que as aguIhas com mandril podem reduzir os riscos de punção do osso sacro, dos vasos e da própria duramáter devido a sua ponta romba. Porém estes riscos podem ser minimizados com as agulhas sem mandril obedecendo aos cuidados de se injetar o anestésico local de modo lento e com freqüentes aspirações. A afirmativa de que agulhas comuns podem ser causa de tumores epidermóides ainda é uma especulação. Não fosse assim estas agulhas não mais constariam da técnica de anestesia caudal em países onde a prática da Medicina está sujeita a freqüentes litígios judiciais. Por isto penso que os que não dispõem de agulhas com mandril deveriam continuar a utilizar as agulhas comuns para realizarem seus bloqueios sacros. Em nosso serviço utilizamos agulhas do tipo butterfly $\mathrm{N}^{\circ \mathrm{s}} 23 \mathrm{G}, 25 \mathrm{G}$ ou $27 \mathrm{G}$, agulhas hipodérmicas ou agu-
Ihas para injeção venosa (tipo Abbocath 22G). Uma alternativa interessante é citada pelo próprio Broadman ${ }^{3}$ : façam um pequeno orifício na pele com uma agulha um pouco mais calibrosa e por ele introduzam a agulha comum.

\section{Cordialmente}

\section{Sérgio Bernardo Tenório, TSA}

Professor Adjunto da Faculdade de Medicina da UFPr Anestesiologista do Hospital Infantil Pequeno Príncipe Rua Dr. Aluízio França 141

80410-710 Curitiba, PR

Email: tenorio@bbs2.sul.com.br

\section{Standard or Mandrel Needles for Pediatric Caudal Block?}

\author{
Mr. Editor,
}

Sacral hiatus puncture for pediatric caudal block has always been performed with standard injection needles and this practice persists in spite of current availability of mandrel needles adequate to pediatric use. Geiduschek ${ }^{1}$ admits that: although some products were especially designed for pediatric regional anesthesia, caudal block may be induced with standard $20 \mathrm{G}$ or $22 \mathrm{G}$ needles or with intravenous catheter. Recently, however, mandrel-less needles for caudal block have been criticized by some authors, such as Dalens ${ }^{2}$, Broadman ${ }^{3}$ and Tobias ${ }^{4}$, for believing that such needles may have epithelial cells inside which, if carried to the spinal cord, could cause epidermoid tumors.

Broadman ${ }^{3}$, in long editorial, mentions 23 references to backup his position against mandrel-less needles for sacral blocks. In my opinion, he has no convincing justifications. He admits, for example, that: a) with a single exception, all epidermoid tumor reports in the literature occurred in the intradural space of patients under spinal anesthesia; $b$ ) there has been no cause/effect relationship between puncture and tumor in all cases; $c$ ) the presence of a mandrel on the needle is no guarantee that it will not have epithelial cells inside, according to a research performed with cadavers; in this study, epithelial cells were found inside 16G Tuohy, 22G Sprotte and $22 \mathrm{G}$ Quincke needles. Similar results were obtained with humans submitted to spinal anesthesia with $25 \mathrm{G}$ needles with mandrel ${ }^{5}$; d) it is still not known whether epidermoid tumors are formed as from needle-transported cells or from embryo cells.

Although some surely well intentioned authorities definitely condemn caudal anesthesia with standard needles, equally outstanding researchers are still using such needles for sacral block. Just to mention some recent publications: a French group has used $22 \mathrm{G}$ needles for caudal block as their single technique for neonates ${ }^{6}$; Canadian anesthesiologists have introduced catheters in the sacral hiatus with intrave- 
nous $18 \mathrm{G}$ needles ${ }^{7}$. The most recent edition of George Gregory's book, world pediatric anesthesia reference, recommends that caudal anesthesia should be induced with hypodermal needles ${ }^{8}$. The same approach is recommended in Morgan et al. ${ }^{9}$ book. Coté and co-editors offer two options: mandrel needles or intravenous puncture needles with catheter ${ }^{10}$.

If there is no consensus in the literature then why don't we change to mandrel needles? For two reasons: mandrel needles cost and availability. Standard needles cost hundreds of times less than mandrel needles and may be found in any hospital. Bradman himself ${ }^{3}$ admits in his editorial that: whenever the size of the patient allows for the use of needles with introducers, they should be used. It is assumed that not even him has mandrel needles for any patient size! So, what to do? To use standard needles or not to induce sacral block are the only alternatives. Would standard needles bring higher risks for the patient than not performing sacral block when indicated?

Caudal anesthesia is extremely useful for children. It is a simple technique, decreasing the need for perioperative anesthetic drugs and muscle relaxants and providing postoperative analgesia. It is certainly not risk-free but there is no risk-free medicine! There is no question that mandrel needles may decrease the risk for sacral bone, vessels and dural puncture due to its blunt point. Those risks, however, may be minimized with mandrel-less needles, if care is taken to slowly inject local anesthetics with frequent aspirations. The statement that standard needles may cause epidermoid tumors is still a speculation. Otherwise, such needles would no longer be part of caudal anesthesia techniques in countries where medical practice is subject to frequent law suits. So, I believe that those not counting on mandrel needles should continue using standard needles for sacral blocks. In our department, we use $23 \mathrm{G}, 25 \mathrm{G}$ or $27 \mathrm{G}$ butterfly needles, hypodermic needles or intravenous injection needles (22G Abbocath). Broadman ${ }^{3}$ himself mentions an interesting alternative: make a small orifice with a larger needle and through it introduce a standard needle.

Yours Truly.

Sérgio Bernardo Tenório, TSA, M.D.

Assistant Professor, Faculdade de Medicina, UFPr

Anesthesiologist, Hospital Infantil Pequeno Príncipe

Rua Dr. Aluízio França 141

80410-710 Curitiba, PR

Email: tenorio@bbs2.sul.com.br

\section{REFERÊNCIAS - REFERENCES}

01. Geiduschek JM - Pediatrics, em: Brown DL - Regional Anesthesia and Analgesia, WB Saunders Company, Philadelphia, 1996;562

02. Dalens BK - Regional Anesthetics Techniques, em: Dalens B, Bissonnette B - Pediatric Anesthesia. Principles \& Practice. New York, Mc Graw-Hill, 2002;536

03. Broadman IM - Where should advocacy for pediatric patients end and concerns for patient safety begin? Reg Anesth, 1997;22:205-208

04. Tobias DJ - New insights into regional anesthesia in children: new techniques and new indications. Current Opinion in Anesthesiology, 2001;14:345-352

05. Campbell DC, Douglas MJ, Taylor G - Incidence of tissue coring with the 25-gauge Quincke and Whitacre spinal needles. Reg Anesth, 1996;21:582-585

06. Cucchiaro G, De Lagausie P, El-Ghonemi A - Single-dose caudal anesthesia for major intra-abdominal operations in high-risk infants. Anesth Analg, 2001;92:1439-1441

07. Tsui BCH, Seal R, Koller J - Thoracic epidural catheter placement via the caudal approach in infants by using electrocardiographic guidance. Anesth Analg, 2002;95: 326-330

08. Sethna NF, Berde CB, em: Gregory G - Pediatric Regional Anesthesia, Churchill Livingstone, 2002;281

09. Kleinmam W - Spinal, Epidural and Caudal Blocks, em: Morgan EGJ, Mikhail MS, Murray MJ - Clinical Anesthesiology, New York, Lange Medical Books, 2002;273

10. Polaner DM, Suresh S, Coté CJ - Pediatric Regional Anesthesia, em: Coté CJ et al - A Practice of Anesthesia for Infants and Children, $3^{\text {rd }}$ Ed, WB Saunders Company, Philadephia; 2001;647 